\title{
ON THE ADJOINT GROUP OF SOME RADICAL RINGS by OLIVER DICKENSCHIED
}

\author{
(Received 18 July, 1995)
}

1. Introduction. A ring $R$ is called radical if it coincides with its Jacobson radical, which means that $R$ forms a group under the operation $a \circ b=a+b+a b$ for all $a$ and $b$ in $R$. This group is called the adjoint group $R^{\circ}$ of $R$. The relation between the adjoint group $R^{\circ}$ and the additive group $R^{+}$of a radical ring $R$ is an interesting topic to study. It has been shown in [1] that the finiteness conditions "minimax", "finite Prüfer rank", "finite abelian subgroup rank" and "finite torsionfree rank" carry over from the adjoint group to the additive group of a radical ring. The converse is true for the minimax condition, while it fails for all the other above finiteness conditions by an example due to Sysak [6] (see also [2, Theorem 6.1.2]). However, we will show that the converse holds if we restrict to the class of nil rings, i.e. the rings $R$ such that for any $a \in R$ there exists an $n=n(a)$ with $a^{n}=0$.

Recall that a group $G$ is called a minimax group if it has a series of finite length whose factors satisfy the minimum or maximum condition on subgroups. The group $G$ has finite torsion-free rank if it has a finite series whose factors are either periodic or infinite cyclic. The number of infinite cyclic factors in any such series is an invariant of $G$ denoted by $r_{0}(G)$. The group $G$ has finite abelian subgroup rank if each abelian subgroup of $G$ has finite torsion-free rank and each abelian $p$-subgroup of $G$ has finite Prüfer rank for every prime $p$. Here a group $G$ is said to have finite Prüfer rank $r=r(G)$ if every finitely generated subgroup of $G$ can be generated by $r$ elements, and $r$ is the least positive integer with this property. For the relation between these finiteness conditions see Chapter 6.3 of [4].

THEOREM A. Let $R$ be a nil ring. Then the following hold.

(a) If $R^{+}$has finite torsion-free rank $n$, then also $r_{0}\left(R^{\circ}\right)=n$.

(b) If $R^{+}$has finite abelian subgroup rank, then so does $R^{\circ}$.

(c) If $R^{+}$has finite Prüfer rank, then so does $R^{\circ}$, and $r\left(R^{\circ}\right) \leq 3 . r\left(R^{+}\right)$. If $R^{+}$contains no elements of order 2 then even $r\left(R^{\circ}\right) \leq 2 . r\left(R^{+}\right)$.

The situation for the class of radical rings with a periodic additive group is similar, as the following result shows.

THEOREM B. Let the additive group $R^{+}$of the radical ring $R$ be periodic. Then the following hold.

(a) If $R^{+}$has finite abelian subgroup rank, then so does $R^{\circ}$.

(b) If $R^{+}$has finite Prüfer rank, then so does $R^{\circ}$, and $r\left(R^{\circ}\right) \leq 3 . r\left(R^{+}\right)$. If $R^{+}$contains no elements of order 2 then even $r\left(R^{\circ}\right) \leq 2 . r\left(R^{+}\right)$.

At the end of Section 2, an example of a radical ring $R$ with $R^{+}$being an elementary abelian $p$-group shows that in the situation of Theorem $\mathrm{B}$, the adjoint group $R^{\circ}$ may have infinite torsion-free rank. The rank inequalities in part (c) of Theorem $A$ and part (b) of Theorem $\mathrm{B}$ depend on the following proposition.

$$
\text { Glasgow Math. J. } 39 \text { (1997) 35-41. }
$$


Proposition. Let $R$ be a nil ring and $n$ a positive integer. If $S=n R$, then $n S=\left(S^{\circ}\right)^{\{n\}}=\left(S^{\circ}\right)^{n}$.

Here $G^{\{n\}}$ denotes the set of all $n$th powers of the elements of a group $G$ and $G^{n}$ the subgroup of $G$ generated by this set.

It seems to be unknown whether the bounds in part (c) of Theorem A and part (b) of Theorem $\mathrm{B}$ are best possible. This question will be discussed in more detail at the end of Section 3.

The notation is standard and can for instance be found in [4] and [5]. Note that the adjoint inverse of an element $a$ of a radical ring will be denoted by $a^{\prime}$.

2. Proof of the proposition and Theorem B. The following is a technical lemma on formal power series.

LEMMA 2.1. Let $\mathbb{Z} \llbracket x \rrbracket$ be the ring of formal power series in the variable $x$ over the ring $\mathbb{Z}$ of integers. If $n$ is a positive integer, then $1+n^{2} x$ can be written as $(1+n . f)^{n}$ for some $f \in x \mathbb{Z} \llbracket x \rrbracket$.

Proof. Considering the binomial series for $\left(1+n^{2} x\right)^{1 / n}$, we obtain that

$$
f=\sum_{m=1}^{\infty} n^{2 m-1}\left(\begin{array}{c}
1 / n \\
m
\end{array}\right) x^{m} \in x \mathbb{R} \llbracket x \rrbracket
$$

satisfies $1+n^{2} x=(1+n f)^{n}$. Thus it suffices to show that

$$
n^{2 m-1}\left(\begin{array}{c}
1 / n \\
m
\end{array}\right)=n^{m-1} \frac{1(1-n)(1-2 n) \ldots(1-(m-1) n)}{m !}
$$

is an integer for $n, m \geq 1$. If $p$ is a prime, then the number of times that $p$ divides $m$ ! is

$$
v_{m}=\sum_{i \geq 1}\left[\frac{m}{p^{i}}\right]
$$

where $[x]$ denotes the greatest integer not exceeding the real number $x$. Hence we only need to show that $p$ divides $n^{m-1} 1(1-n)(1-2 n) \ldots(1-(m-1) n)$ at least $v_{m}$ times. As

$$
v_{m}<\frac{m}{p} \sum_{i=0}^{\infty}\left(\frac{1}{p}\right)^{i}=\frac{m}{p-1} \leq m,
$$

this is clear if $p$ divides $n$. Therefore we may suppose that $p$ does not divide $n$. Then at least $\left[\frac{m}{p^{i}}\right]$ of the factors

$$
1,1-n, 1-2 n, \ldots, 1-(m-1) n
$$

are divisible by $p^{i}$ for every $i \geq 1$, from which it follows that $p$ divides the product $1(1-n)(1-2 n) \ldots(1-(m-1) n)$ at least $v_{m}$ times. This completes the proof of the lemma. 
Proof of the proposition. As $n S$ is an ideal of $R$, it forms a subgroup of the adjoint group $R^{\circ}$. Thus we only have to show that $n S=\left(S^{\circ}\right)^{\{n\}}$.

Let $t \in n S$, i.e. $t=n s$ for some $s \in S$. Then $s=n r$ for a suitable $r \in R$. By Lemma 2.1 there exists a formal power series $f \in x \mathbb{Z} \llbracket x \rrbracket$ such that $(1+n f)^{n}=1+n^{2} x$. Putting $a=n . f(r) \in n R=S$ and using a formal identity 1 , we obtain $(1+a)^{n}=1+n^{2} r=1+t$. Note that the substitution of $r$ into $f$ is possible, since $R$ is nil. It follows that $t$ is the adjoint $n$th power of $a \in S$, which implies $n S \subseteq\left(S^{\circ}\right)^{\{n\}}$.

Now let $p$ be a prime dividing $n$. If $s \in S$, then $s=p r$ for some $r \in R$. It follows that

$$
(1+s)^{p}=1+\sum_{i=1}^{p-1}\left(\begin{array}{c}
p \\
i
\end{array}\right) s^{i}+s^{p}
$$

where

and

$$
\sum_{i=1}^{p-1}\left(\begin{array}{c}
p \\
i
\end{array}\right) s^{i}=p s \cdot \sum_{i=1}^{p-1} \frac{1}{p}\left(\begin{array}{c}
p \\
i
\end{array}\right) s^{i-1} \in p S
$$

$$
s^{p}=p^{p} r^{p}=p^{p-2} r^{p-1} \cdot p s \in p S .
$$

Hence we have $(1+S)^{\{p\}} \subseteq 1+p S$. Writing $n=p_{1} \ldots p_{k}$ as a product of primes, it now follows by induction on $k$ that $(1+S)^{\{n\}} \subseteq 1+n S$. Thus $\left(S^{\circ}\right)^{\{n\}} \subseteq n S$. The proposition is proved.

To apply the proposition for radical $p$-rings recall that a finite $p$-group $G$ is called powerful if either $p=2$ and $G^{\prime} \leq G^{4}$ or $p$ is an odd prime and $G^{\prime} \leq G^{p}$. Writing $d(G)$ for the minimal number of elements from $G$ necessary to generate $G$, we have the following facts, which can for instance be found in [3].

Lemma 2.2. Let $G$ be a finite p-group.

(a) (Burnside Basis Theorem.) If $\Phi(G)$ denotes the Frattini subgroup of $G$, then $p^{d(G)}=|G / \Phi(G)|$.

(b) If $G$ is powerful, then $r(G)=d(G)$ and $\Phi(G)=G^{p}$.

LEMMA 2.3. Let $R$ be a finite nilpotent $p$-ring.

(a) If $p=2$, then $r\left((4 R)^{\circ}\right)=r\left((4 R)^{+}\right)$.

(b) If $p$ is an odd prime, then $r\left((p R)^{\circ}\right)=r\left((p R)^{+}\right)$.

Proof. Let $S=n R$, where $n=4$ if $p=2$, and $n=p$ if $p$ is odd. For all $x, y \in S$, the adjoint commutator $x^{\prime} \circ y^{\prime} \circ x \circ y=\left(1+x^{\prime}\right)\left(1+y^{\prime}\right)(x y-y x)$ lies in $S^{2}=n^{2} R^{2} \subseteq n^{2} R=n S$. Hence it follows from the proposition that $\left(S^{\circ}\right)^{\prime} \subseteq\left(S^{\circ}\right)^{n}$. Thus $S^{\circ}$ is powerful. Now Lemma 2.2 and again the proposition yield

$$
p^{r\left(S^{\circ}\right)}=p^{d\left(S^{\circ}\right)}=\left|S^{\circ} / \Phi\left(S^{\circ}\right)\right|=\left|S^{\circ} /\left(S^{\circ}\right)^{p}\right|=\left|S^{+} /(p S)^{+}\right|=p^{r\left(S^{+}\right)} .
$$

This proves the lemma.

As a consequence, part (b) of Theorem B follows for radical $p$-rings.

LeMmA 2.4. Let $R$ be a radical $p$-ring whose additive group $R^{+}$has finite Prüfer rank. Then $R^{\circ}$ has likewise finite Prüfer rank and the following hold.

(a) If $p=2$, then $r\left(R^{\circ}\right) \leq 3 . r\left(R^{+}\right)$. 
(b) If $p$ is an odd prime, then $r\left(R^{\circ}\right) \leq 2 \cdot r\left(R^{+}\right)$.

Proof. Consider first the case that $R$ is finite. Then $R$ is nilpotent; see [5, Theorem 2.5.16]. For any subgroup $U$ of $(R / p R)^{\circ}$, it follows by the Burnside Basis Theorem that

$$
p^{d(U)}=|U / \Phi(U)| \leq|R / p R|=p^{r\left((R / p R)^{+}\right)} .
$$

Hence

$$
r\left((R / p R)^{\circ}\right)=\max _{U \leq(R / p R)^{\circ}} d(U) \leq r\left((R / p R)^{+}\right) \leq r\left(R^{+}\right) .
$$

In case (b), Lemma 2.3 yields

$$
r\left((p R)^{\circ}\right)=r\left((p R)^{+}\right)
$$

from which it follows that

$$
r\left(R^{\circ}\right) \leq r\left(R^{\circ} /(p R)^{\circ}\right)+r\left((p R)^{\circ}\right)=r\left((R / p R)^{\circ}\right)+r\left((p R)^{\circ}\right) \leq 2 . r\left(R^{+}\right) .
$$

Case (a) is treated in the same way by considering the chain

$$
0 \leq 4 R \leq 2 R \leq R
$$

and observing that the ring $2 R / 4 R$ has trivial multiplication, so that its additive and adjoint groups coincide.

Consider now the general case of an arbitrary radical $p$-ring. For all $n \geq 0$ let $R_{n}$ be the ideal $\left\{r \in R \mid p^{n} r=0\right\}$ of $R$. As $R$ is a $p$-ring, we have

$$
R=\bigcup_{n \geq 0} R_{n} \text {. }
$$

Let $U$ be a finitely generated subgroup of $R_{n}^{+}$. Then $U$ is an $r$-generated abelian group of exponent dividing $p^{n}$, where $r=r\left(R^{+}\right)$. Thus $|U| \leq\left(p^{n}\right)^{r}=p^{n r}$. Hence each $R_{n}$ is finite. Let $c=3$ for $p=2$ and $c=2$ for $p \neq 2$. By the finite case we have

$$
r\left(R_{n}^{\circ}\right) \leq c . r\left(R_{n}^{+}\right) \leq c \cdot r\left(R^{+}\right)
$$

for all $n \geq 0$. Since $R^{\circ}$ is the union of the $R_{n}^{\circ}$, we obtain

$$
r\left(R^{\circ}\right) \leq c \cdot r\left(R^{+}\right) \text {. }
$$

The lemma is proved.

To complete the proofs of both of the theorems we will need the following result.

Lemma 2.5 ([1, Lemma 2.4]). If $R$ is a nil ring and $p$ a prime, then the following hold.

(a) $R^{+}$is a $p$-group if and only if $R^{\circ}$ is a p-group.

(b) $R^{+}$is torsion-free if and only if $R^{\circ}$ is torsion-free.

Proof of Theorem B. To prove part (a), suppose that $R^{+}$is periodic with finite abelian subgroup rank. For each prime $p$ the $p$-component of $R^{+}$forms an ideal $T_{p}$ of the ring $R$, and $R=\underset{p}{\oplus} T_{p}$. By [4, Vol. 2, p. 38, Corollary 1], each $T_{p}^{+}$is a Chernikov-group. Hence by Theorem A of [1] , each $T_{p}^{\circ}$ is a Chernikov-group and each of the ideals $T_{p}$ of $R$ is nilpotent. In particular, each $T_{p}^{\circ}$ is a nilpotent group. As $R^{\circ}$ is the direct product of the $T_{p}^{\circ}$, it follows that $R^{\circ}$ is locally nilpotent (i.e. each of its finitely generated subgroups is nilpotent). By the nilpotency of the rings $T_{p}$ and Lemma 2.5 each $T_{p}^{\circ}$ is a 
Chernikov $p$-group. Thus $R^{\circ}=\underset{p}{\otimes} T_{p}^{\circ}$ has finite abelian subgroup rank by [4, Vol. 2, p. 38, Corollary 1].

Part (b) is proved in the same way, using [4, Vol. 2, p. 38, Corollary 2]. Here, by Lemma 2.4, the bound $r=r\left(R^{+}\right)$for the Prüfer ranks of the $T_{p}^{+}$carries over to the required bound for the Prüfer ranks of the $T_{p}^{\circ}$.

We finish this section with the example mentioned in the introduction.

EXAMPLE (see $[6$, p. 28]). Let $p$ be a prime and $K=\mathrm{GF}(p)$ the field with $p$ elements. If $K \llbracket x \rrbracket$ denotes the ring of formal power series over $K$ then $R=x K \llbracket x \rrbracket$ is a radical ring with an elementary abelian additive group $R^{+}$. But obviously the element $x$ of $R^{\circ}$ has infinite order in $R^{\circ}$. (In fact, it can easily be shown that $R^{\circ}$ is torsion-free.) Assume $r_{0}\left(R^{\circ}\right)<\infty$. Then Theorem B of [1] implies that $r_{0}\left(R^{\circ}\right)=r_{0}\left(R^{+}\right)=0$, contradicting the fact that $R^{\circ}$ contains elements of infinite order. Hence $r_{0}\left(R^{\circ}\right)=\infty$.

3. Proof of Theorem A. A ring $R$ is called locally nilpotent if each of its finitely generated subrings is nilpotent.

LeMma 3.1 ([1, Lemma 2.1]). Let $R$ be a nilpotent ring and $¥$ a class of groups which is closed under the forming of subgroups, epimorphic images and extensions. Then the adjoint group $R^{\circ}$ of $R$ is an $\mathfrak{X}$-group if and only if the additive group $R^{+}$of $R$ is an $\mathfrak{x}$-group.

LEMMA 3.2 ([1, Lemma 3.1]). If $R$ is a locally nilpotent ring whose additive group $R^{+}$ is torsion-free with finite torsion-free rank $n$, then $R^{n+1}=0$.

Lemma 3.3 (Special case of [7, Theorem 6]). Let $R$ be an arbitrary ring and $S$ a nilpotent proper subring of $R$. Then $S$ is properly contained in its idealizer $\operatorname{Id}_{R}(S)=$ $\{r \in R \mid r S+S r \subseteq S\}$.

LEMMA 3.4. Let $G$ be a locally nilpotent torsion-free group with finite torsion-free rank. Then $r(G) \leq r_{0}(G)<\infty$.

Proof. We may assume that $G$ is finitely generated and hence nilpotent. Let

$$
1=Z_{1} \leq Z_{2} \leq \ldots \leq Z_{n}=G
$$

be the upper central series of $G$. As $Z_{1}$ is torsion-free, each of the factors $Z_{i+1} / Z_{i}$ for $i<n$ is torsion-free abelian; see [4, Vol. 1, Theorem 2.25]. Thus

$$
r(G) \leq \sum_{i=1}^{n-1} r\left(Z_{i+1} / Z_{i}\right)=\sum_{i=1}^{n-1} r_{0}\left(Z_{i+1} / Z_{i}\right)=r_{0}(G)
$$

The lemma is proved.

Proof of Theorem $A$. The torsion subgroup of $R^{+}$forms an ideal $T$ of $R$. If the ideals $T_{p}$ of $R$ are defined as in the proof of Theorem B, then $T=\oplus T_{p}$. By Lemma 2.5, each $T_{p}^{\circ}$ is a $p$-group and $(R / T)^{\circ}$ is torsion-free.

To prove (a), note that $T^{\circ}=\otimes T_{p}^{\circ}$ is periodic, so that we may assume $T=0$. Hence $R^{+}$is torsion-free. By Zorn's Lemma there exists a maximal locally nilpotent subring $S$ of $R$, which is even nilpotent by Lemma 3.2. Assume now that $S \neq R$. Then by Lemma 3.3, $S$ is properly contained in its idealizer $I=\operatorname{Id}_{R}(S)$. Hence there exists an element $a$ in the 
subring $l$ of $R$ which is not in $S$. The subring $\hat{S}$ generated by $S \cup\{a\}$ is contained in the idealizer $I$ of $S$, and therefore $S$ is an ideal of $\hat{S}$. The quotient ring $\hat{S} / S$ is generated by $a+S$. As $R$ is nil, it follows that $\hat{S} / S$ is nilpotent. Thus $\hat{S}$ is a nilpotent subring of $R$ containing $S$ properly. This contradiction shows that $R=S$ is nilpotent. Now Lemma 3.1 yields $r_{0}\left(R^{\circ}\right)<\infty$, so that Theorem B of [1] implies $r_{0}\left(R^{\circ}\right)=r_{0}\left(R^{+}\right)$. This proves part (a).

To prove (b), let $R^{+}$have finite abelian subgroup rank. Then $T^{+}$and hence by Theorem B also $T^{\circ}$ have finite abelian subgroup rank. Moreover, $T$ is a locally nilpotent ring, since we have seen in the proof of Theorem $\mathrm{B}$, that $T$ is the direct sum of the nilpotent ideals $T_{p}$. As $r_{0}\left((R / T)^{+}\right)<\infty$, it follows as in the proof of (a) that $R / T$ is a nilpotent ring. Its additive group $(R / T)^{+}$is torsion-free with finite abelian subgroup rank and hence has finite Prüfer rank by $[4$, Vol. 2, p. 38, Corollary 1]. Thus Lemma 3.1 implies $r\left((R / T)^{\circ}\right)<\propto$ and, in particular, $(R / T)^{\circ}$ has finite abelian subgroup rank. As $R / T$ is nilpotent and $T$ is locally nilpotent, the ring $R$ is locally nilpotent. Thus $R^{\circ}$ is a locally nilpotent extension of $T^{\circ}$ by $(R / T)^{\circ}$, which both have finite abelian subgroup rank. Hence $R^{\circ}$ has finite abelian subgroup rank. This proves (b).

To prove (c), suppose that $R^{+}$has finite Pruifer rank. Then it follows as in the proof of (b) that $R$ is a locally nilpotent ring and that $R^{\circ}$ is a locally nilpotent group. Moreover,

$$
r\left((R / T)^{\circ}\right) \leq r_{0}\left((R / T)^{\circ}\right)=r_{0}\left((R / T)^{+}\right)
$$

by Lemma 3.4 and part (a). On the other hand, Theorem $B$ yields

$$
r\left(T^{\circ}\right) \leq c \cdot r\left(T^{+}\right)<\infty
$$

where $c=2$ if $R$ contains no elements of order 2 and otherwise $c=3$. Combining equations (1) and (2), we obtain

$$
\begin{aligned}
r\left(R^{\circ}\right) & \leq r\left(R^{\circ} / T^{\circ}\right)+r\left(T^{\circ}\right)=r\left((R / T)^{\circ}\right)+r\left(T^{\circ}\right) \\
& \leq r_{0}\left((R / T)^{+}\right)+c \cdot r\left(T^{+}\right) \\
& \leq c \cdot\left(r_{0}\left(R^{+} / T^{+}\right)+r\left(T^{+}\right)\right) \\
& =c \cdot r\left(R^{+}\right) .
\end{aligned}
$$

This completes the proof of Theorem A.

REMARKs. (a) Note that our main results together with Theorem B of [1] imply that the rings $R$ considered in the theorems with $R^{+}$having finite abelian subgroup rank are two-sided $T$-nilpotent, i.e. each non-trivial epimorphic image of $R$ has a non-trivial two-sided annihilator. It is easy to see that such rings are locally nilpotent.

(b) In both theorems, the inequality $r\left(R^{\circ}\right) \leq c . r\left(R^{+}\right)$for the Prüfer ranks is given, where $c=2$ if $R^{+}$contains no elements of order 2 and otherwise $c=3$. It remains open whether these bounds are best possible. For rings with elements of additive order 2 , the worst case known to the author is the ring $R=2 \mathbb{Z} / 8 \mathbb{Z}$ with $r\left(R^{+}\right)=1$ and $r\left(R^{\circ}\right)=2$, while in the special case in which $R^{+}$contains no elements of order 2, no example $R$ with $r\left(R^{\circ}\right)>r\left(R^{+}\right)$seems to be known. Hence it can be conjectured that the constant $c$ can be decreased by 1 in either case.

(c) A slight modification of our proofs leads to the following minor improvement of the inequality just discussed:

$$
r\left(R^{\circ}\right) \leq r_{0}\left(R^{+}\right)+\max \left\{3 \cdot r\left(T_{2}^{+}\right), 2 \cdot r\left(T_{p}^{+}\right) \mid p \neq 2\right\}
$$

where the $T_{p}$ are defined as in the proof of Theorem B. 
ACKNOWLEDGEMENT. The author would like to thank the referee for considerably shortening the proof of Lemma 2.1 .

\section{REFERENCES}

1. B. Amberg and O. Dickenschied, On the adjoint group of a radical ring, Canad. Math. Bull. 38 (1995), 262-270.

2. B. Amberg, S. Franciosi and F. de Giovanni, Products of groups (Oxford University Press, 1992).

3. J. D. Dixon, M. P. F. du Sautoy, A. Mann and D. Segal, Analytic pro-p-groups (Cambridge University Press, 1991).

4. D. J. S. Robinson, Finiteness conditions and generalized soluble groups (Springer, 1972).

5. L. H. Rowen, Ring theory (Academic Press, 1988).

6. Ya. P, Sysak, Products of infinite groups, Akad. Nauk. Ukrain. SSR Inst. Mat. Preprint 82.53 (1982), (in Russian).

7. F. Szász, On the Idealizer of a subring, Monatsh. Math. 75 (1971), 65-68.

FaCHBEREICH MathematiK

DER UNIVERSITÄT MAINZ

D-55099 MAINZ

E-mail: dickenschied@mat.mathematik.uni-mainz.de 